Article

\title{
UPEI-400, a Conjugate of Lipoic Acid and Scopoletin, Mediates Neuroprotection in a Rat Model of Ischemia/Reperfusion
}

\author{
Barry J. Connell ${ }^{1}$, Monique C. Saleh ${ }^{1}$, Desikan Rajagopal ${ }^{2}$ and Tarek M. Saleh ${ }^{1,3, *}$
}

1 Department of Biomedical Science, Atlantic Veterinary College, University of Prince Edward Island, Charlottetown, PE C1A 4P3, Canada; connell@upei.ca (B.J.C.); msaleh@upei.ca (M.C.S.)

2 Department of Chemistry \& Pharmaceutical Chemistry, School of Advanced Sciences, VIT University, Vellore 632014, India; desikanrajagopal@gmail.com

3 Department of Biomedical Science, Ontario Veterinary College, University of Guelph, Guelph, ON N1G 2W1, Canada

* Correspondence: tsaleh@uoguelph.ca

Short Title: UPEI-400 mediates neuroprotection following ischemia/reperfusion 


\begin{abstract}
1) Background: Previously, our laboratory has provided evidence that pre-administration of the antioxidant, lipoic acid covalently bonded to various naturally occurring antioxidants, enhanced neuroprotective capacity compared to the administration of lipoic acid on its own. The naturally occurring compound scopoletin, a coumarin derivative, has been shown in various in vitro studies to have both antioxidant and anti-inflammatory mechanism of actions. To date, the effect of scopoletin on neuronal cell death in an in vivo model of ischemia or ischemia-reperfusion has not been investigated. Therefore, the present investigation was designed to determine if scopoletin on its own, or a co-drug consisting of lipoic acid and scopoletin covalent bond, named UPEI-400, would be capable of demonstrating a similar neuroprotective efficacy.
\end{abstract}

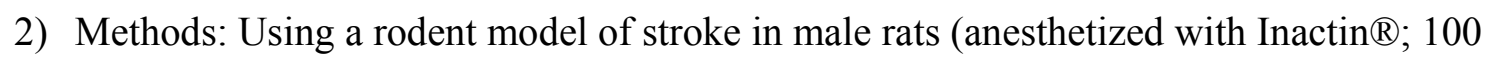
$\mathrm{mg} / \mathrm{kg}$, iv), the middle cerebral artery was permanently occluded for 6 hours (pMCAO), or in separate animals, occluded for $30 \mathrm{~min}$ followed by $5.5 \mathrm{hrs}$ of reperfusion (ischemia/reperfusion; $\mathrm{I} / \mathrm{R}$ ).

3) Results: Pre-administration of either scopoletin or UPEI-400 significantly decreased infarct volume in the I/R model $(\mathrm{p}<0.05)$, but not in the pMCAO model of stroke. However, UPEI-400 was $\sim 1000$ times more potent as compared to scopoletin on its own. The optimal dose of UPEI-400 was then injected during the occlusion and at several time points during reperfusion and significant neuroprotection was observed for up to 150 mins following the start of reperfusion $(\mathrm{p}<0.05)$.

4) Conclusion: The data suggest that synthetic combination of scopoletin with lipoic acid (UPEI-400) is a more effective neuroprotectant that either compound on their own. Also, since UPEI-400 was only effective in a model of $I / R$, it is possible that it may act to 
enhance neuronal antioxidant capacity and/or upregulate anti-inflammatory pathways to prevent the neuronal cell death.

Keywords: stroke; antioxidant; co-drug; animal model

\section{INTRODUCTION}

According to the most recent published data in 2016, death and disability due to stroke remains a global medical issue and therapeutic options remain limited [1]. Ischemic stroke, resulting from the occlusion of a cerebral artery, accounts for up to $80 \%$ of all strokes [2]. A transient or permanent reduction of cerebral blood flow results in hypoxia, hypoglycemia, the failure of ATP-dependent pumps and a disruption of ionic equilibrium, leading to the generation of reactive oxygen species (ROS) and free radicals, and the upregulation of cytokine inflammatory pathways, ultimately ending in neuronal damage and death $[2,3]$. Research into antioxidant and anti-inflammatory therapy to prevent and/or scavenge ROS and free radical generation has provided hope that the damage due to reperfusion injury may be mitigated, and that the window of opportunity for therapeutic treatment, such as tissue-plasminogen-activator (TPA), may be extended beyond the currently accepted time frame of 3 to 4 hours following the onset of stroke.

Scopoletin (6-methoxy-7-hydroxycoumarin) is a coumarin derivative found in various plants and has a long history of use for its medicinal properties in traditional Chinese medicine [4]. Scopoletin is isolated from Canarium patentinervium Miq (Burseraceae Kunth), a rare plant from the family of Burseraceae and genus Canarium found in the Asia-Pacific region and has been used in wound healing by the indigenous people of Malaysia [5]. In particular, evidence has been provided to suggest that scopoletin has both antioxidant and anti-inflammatory 
properties and has been used to ameliorate the symptoms of various inflammatory, rheumatoid and digestive disorders [4]. In vitro studies have demonstrated that scopoletin has antioxidant properties, is an effective inhibitor of the activation of 5-lipoxygenase and has antiacetylcholinesterase activity [6]. To date, scopoletin has not been tested in any in vivo model of stroke (ischemia or ischemia/reperfusion), but other coumarin derivatives have been tested with some success $[7,8]$.

Clinically, single drug therapies following stroke have not yielded the positive clinical trial results expected when compared to the laboratory testing of these compounds. Due to the multifactorial nature of ischemia and reperfusion injury, it is likely that a particular drug will be unable to affect several pathways simultaneously. Thus, there has been a recent interest in the use of co-drugs as a therapeutic approach in various pathologies $[9,10]$. The development and administration of a co-drug, containing lipoic acid (LA) covalently linked to another compound, have been demonstrated to have a greater efficacy/potency compared to the administration of a mixture of the two drugs $[11,12,13,14,15,16]$. The antioxidant LA, is a known free radical scavenger $[17,18]$, and many researchers have shown that in several different stroke models administration of lipoic acid on its own can be neuroprotective but only at relatively high doses $[19,20,21,22,23,24]$. Further, combining lipoic acid with other drugs has been shown to produce an additive or synergistic protective effect in several different animal models of pathology $[25,26,27,28,29,30,31]$, when compared to the effect of either drug alone. Our laboratory has demonstrated that LA covalently bonded to various naturally occurring antioxidant compounds has provided superior neuroprotection following I/R injury compared to either drug alone or a mixture of 2 compounds administered simultaneously [11,12,13,14,32]. Based on the above observations demonstrating the beneficial effects of developing a co-drug, our laboratory has 
developed a new synthetic co-drug that is a covalent conjugate between LA and scopoletin, named UPEI-400.

In the present study, using a model of acute stroke and reperfusion injury (I/R) in male rats developed in our laboratory [33], we determined whether the administration of scopoletin alone is neuroprotective, and if the administration of UPEI-400 would provide a further improvement of the neuroprotection observed with scopoletin alone. Further, a neuroprotective dose of UPEI-400 was then tested at various time points following reperfusion to examine the neuroprotective capability of UPEI-400 in a more clinically relevant paradigm.

\section{MATERIALS and METHODS}

\section{Ethics statement}

All experiments were carried out in accordance with the guidelines of the Canadian Council on Animal Care and were approved by the University of Prince Edward Island Animal Care Committee (protocol \#11-045 and 13-036).

General surgical procedures for in vivo stroke surgery

All experiments were conducted on male Sprague-Dawley rats (300-350 g; Charles Rivers; Montreal, PQ, CAN). For all animals, food and tap water were available ad libitum. Rats

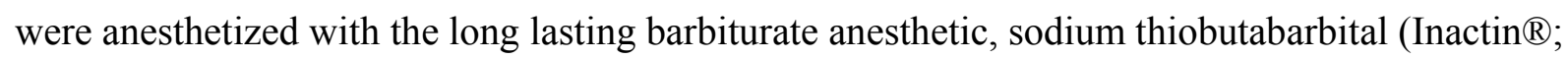
Sigma-Aldridge; St. Louis, MO, USA; $100 \mathrm{mg} / \mathrm{kg}$; ip). Supplements were given if a withdrawal reflex was elicited following corneal or toe pinch every 10 mins. All animals were tracheotomized to facilitate breathing and then placed on a heating blanket to maintain body 
temperature at $37 \pm 1{ }^{\circ} \mathrm{C}$ (Physitemp Instruments; Clifton, NJ, USA).

Transient and permanent middle cerebral artery occlusion (tMCAO and pMCAO)

We have previously published the detailed methodology for occlusion of the middle cerebral artery $[12,13,32,33]$ in both permanent (pMCAO) and transient (tMCAO) models.

\section{UPEI-400 synthesis}

Scopoletin and lipoic acid were chemically linked via an ester bond using a simple synthetic route. Accordingly, scopoletin (0.012 g) was placed in a dry $100 \mathrm{ml}$ flask followed by lipoic acid (0.025M in $0.02 \mathrm{ml}$ of dimethylaminopyridine; DMAP) in $100 \mathrm{ml}$ anhydrous dichloromethane $\left(\mathrm{CH}_{2} \mathrm{Cl}_{2}\right)$. Dicyclo-hexylcarbodiimide (DCC) $(0.025 \mathrm{M})$ was added in small quantities over a period of 1 hour under nitrogen atmosphere. The reaction was stirred overnight and the compound was purified using silica column chromatography after an aqueous work up. Proton $\left({ }^{1} \mathrm{H}\right)$ nuclear magnetic resonance spectroscopy and mass spectrometry was used to characterized the pure compound, UPEI-400.

\section{UPEI -400}

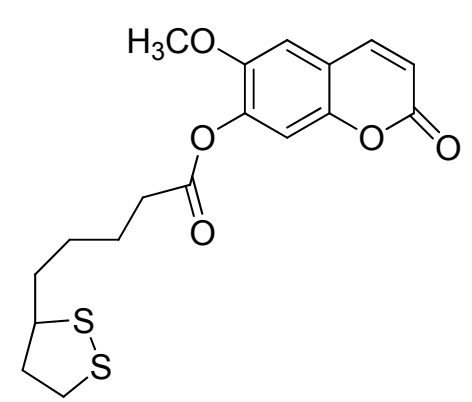

Chemical Formula: $\mathrm{C}_{18} \mathrm{H}_{20} \mathrm{O}_{5} \mathrm{~S}_{2}$

Exact Mass: 380.07522

Molecular Weight: 380.47840 
Preparation of UPEI-400 (synthetic scopoletin-lipoic acid combination)

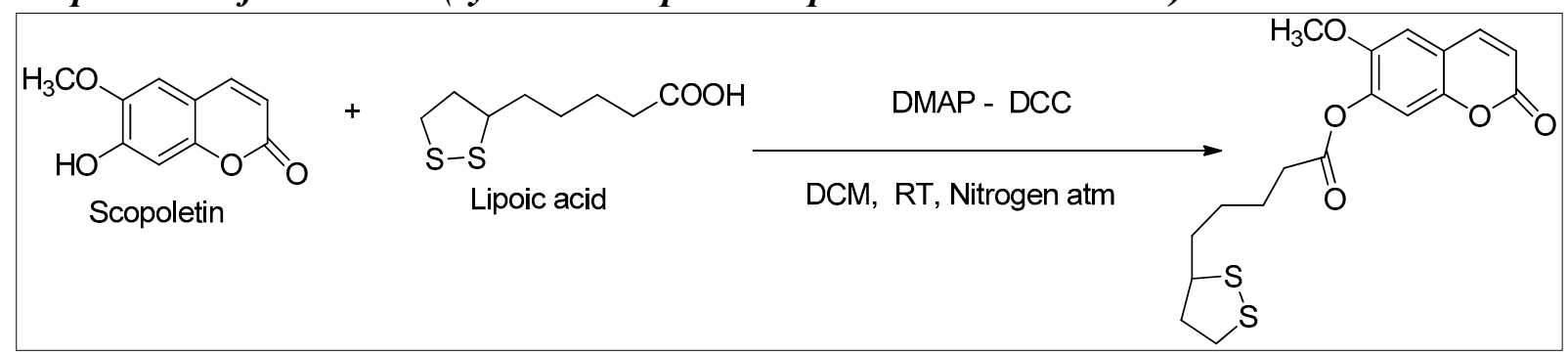

Drug injection protocol in tMCAO model

Scopoletin, UPEI-400 or vehicle were administered ( $1 \mathrm{ml} / \mathrm{kg}$; i.v.) 30 minutes prior to the onset of MCAO (-60 on schematic). The sutures were left in place for 30 minutes (duration of ischemia; MCAO on schematic), and then removed to allow the return of blood flow and reperfusion which lasted for 5.5 hours ( 0 to 330 minutes on the schematic). Post-MCAO administration of UPEI-400 was investigated by administering the lowest dose of UPEI-400 which was previously shown to produce significant neuroprotection at the following time points during the ischemia/reperfusion protocol: 15 minutes prior to the onset of reperfusion (-15), and $30,90,150$ or 210 minutes following the start of reperfusion.

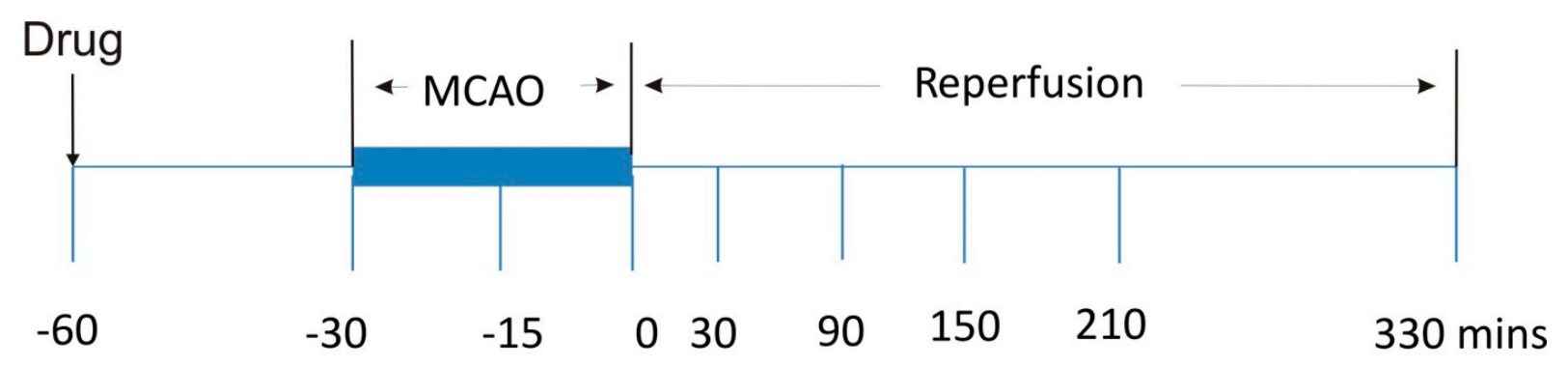

Effect of scopoletin on ischemia-reperfusion (tMCAO model) 
In the first set of animals, scopoletin $(1.0(n=6), 0.1(n=6)$ or $0.01(n=6) \mathrm{mg} / \mathrm{kg})$ was administered in a volume of $1 \mathrm{ml} / \mathrm{kg}$ (i.v.) or vehicle (30\% DMSO; $1 \mathrm{ml} / \mathrm{kg}$; i.v.; $\mathrm{n}=8) 30$ minutes prior to the onset of MCAO (-30 min). Following this $30 \mathrm{~min}$ occlusion, sutures were removed and the region allowed to reperfuse for an additional 5.5 hours.

\section{Effect of UPEI-400 on ischemia-reperfusion (tMCAO model)}

In the second set of experiments, UPEI-400 $(0.1(n=6), 0.01(n=6), 0.001(n=6)$ or 0.0001 $(\mathrm{n}=6) \mathrm{mg} / \mathrm{kg}))$ was administered in a volume of $1 \mathrm{ml} / \mathrm{kg}$ (i.v.) or vehicle $(0.05 \% \mathrm{DMSO} ; 1 \mathrm{ml} / \mathrm{kg}$; i.v.; $\mathrm{n}=6) 30$ minutes prior to the onset of $\mathrm{MCAO}(-30 \mathrm{~min})$. The sutures were left in place for 30 minutes, followed by 5.5 hours of reperfusion.

Effect of UPEI-400 on infarct volume when administered either during the occlusion or reperfusion

To determine if UPEI-400 was neuroprotective when administered during the $30 \mathrm{~min}$ period of occlusion, the optimal dose of UPEI-400, which based on the dose-response-curve described above was $0.001 \mathrm{mg} / \mathrm{kg}$, was administered 15 minutes into the $30 \mathrm{~min}$ occlusion period. The sutures were then removed for an additional 5.5 hours of reperfusion.

To examine the effect of UPEI-400 on reperfusion injury alone, injections of the optimal dose of UPEI-400 (0.001 mg/kg; $1 \mathrm{ml} / \mathrm{kg}$; iv; $\mathrm{n}=5-6$ per group $)$ were made 30, 90, 150 or 210 minutes following suture removal (start of reperfusion). Experiments were then terminated at the end of the 5.5 hours of reperfusion. In all cases, the comparison group far statistical analysis was UPEI-400 vehicle (0.05\% DMSO; $\mathrm{n}=6)$. 
Effect of UPEI-400 on ischemia alone (permanent occlusion; pMCAO)

To determine if administration of UPEI-400 (0.001 mg/kg; iv; $1 \mathrm{ml} / \mathrm{kg} ; \mathrm{n}=5)$ was neuroprotective on ischemia-induced cell death only, injections of UPEI-400 were made 30 minutes prior to pMCAO. Following 6 hours of occlusion, the experiment was terminated. Similar to the studies on tMCAO described above, the vehicle for UPEI-400 tested in the pMCAO model was also $0.05 \%$ DMSO ( $1 \mathrm{ml} / \mathrm{kg}$; iv; $\mathrm{n}=5)$.

\section{Effect of UPEI-400 on baseline blood pressure and heart rate}

To determine the hemodynamic effect of UPEI-400 on baseline (resting) blood pressure and heart rate, continuous recordings of blood pressure and heart rate were taken prior to and for 2 hours following UPEI-400 $(0.001 \mathrm{mg} / \mathrm{kg} ; 1 \mathrm{ml} / \mathrm{kg} ; \mathrm{iv} ; \mathrm{n}=4)$ or vehicle $(0,05 \% \mathrm{DMSO}, 1 \mathrm{ml} / \mathrm{kg}$; iv) administration. Cardiovascular data acquisition and analysis was done as described in our previous studies [33]. Measurements of the blood pressure and heart rate values were made 5 minutes immediately prior to drug administration and at 5, 10, 15, 30, 45, 60, 90 and 120 minutes following drug administration.

\section{Histological Procedures}

At the end of each experiment where infarct volume was measured, animals were overdosed with Inactin $(100 \mathrm{mg} / \mathrm{kg})$, diaphragm transected and left ventricle of the heart perfused with phosphate buffered saline (PBS 0.1 M; $200 \mathrm{ml}$ ) via an 18-gauge needle. The brains were removed and infarct volumes measured as previously described [12,13,32,33]. Digital images were coded prior to being sent for analysis of infarct volume to allow the analyst to be blinded to treatment groups. 


\section{Statistical analysis}

All data was analyzed using SigmaStat and SigmaPlot (Jandel Scientific, Tujunga, CA). Data are presented as a mean \pm standard error of the mean (S.E.M). Using an analysis of variance (ANOVA) followed by a Bonferroni posthoc analysis, differences were considered significant if $\mathrm{p} \leq 0.05$. If only two groups were being compared, the Student's t-test was used.

\section{RESULTS}

The effect of pre-administration of scopoletin on ischemia-reperfusion (tMCAO)

Pre-administration of scopoletin resulted in a dose-dependent neuroprotection with 1.0 $\mathrm{mg} / \mathrm{kg}$ resulting in a significant decrease in infarct volume compared to the administration of vehicle ( $\mathrm{p} \leq 0.05$; Fig.1A and 1B). 
A

UPEI-400 $(0.001 \mathrm{mg} / \mathrm{kg})$

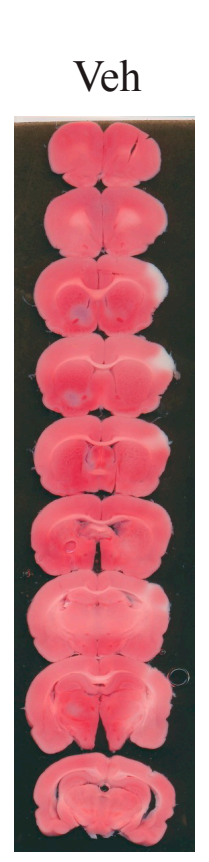

$-15$

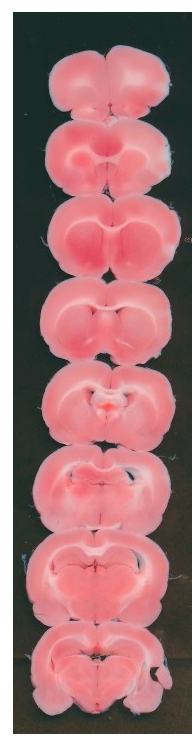

30
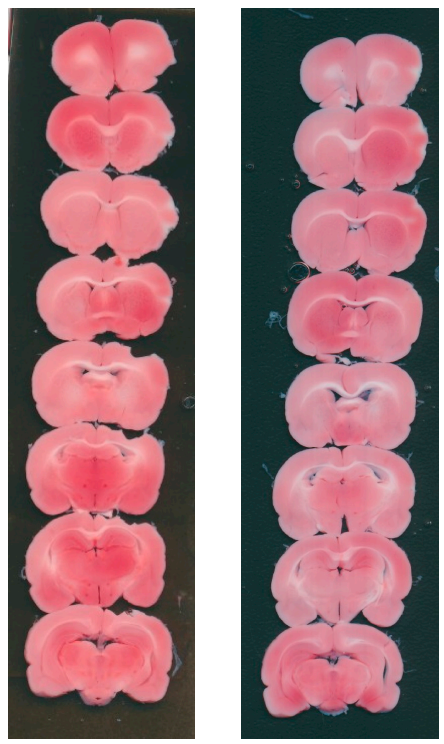

150

210

B

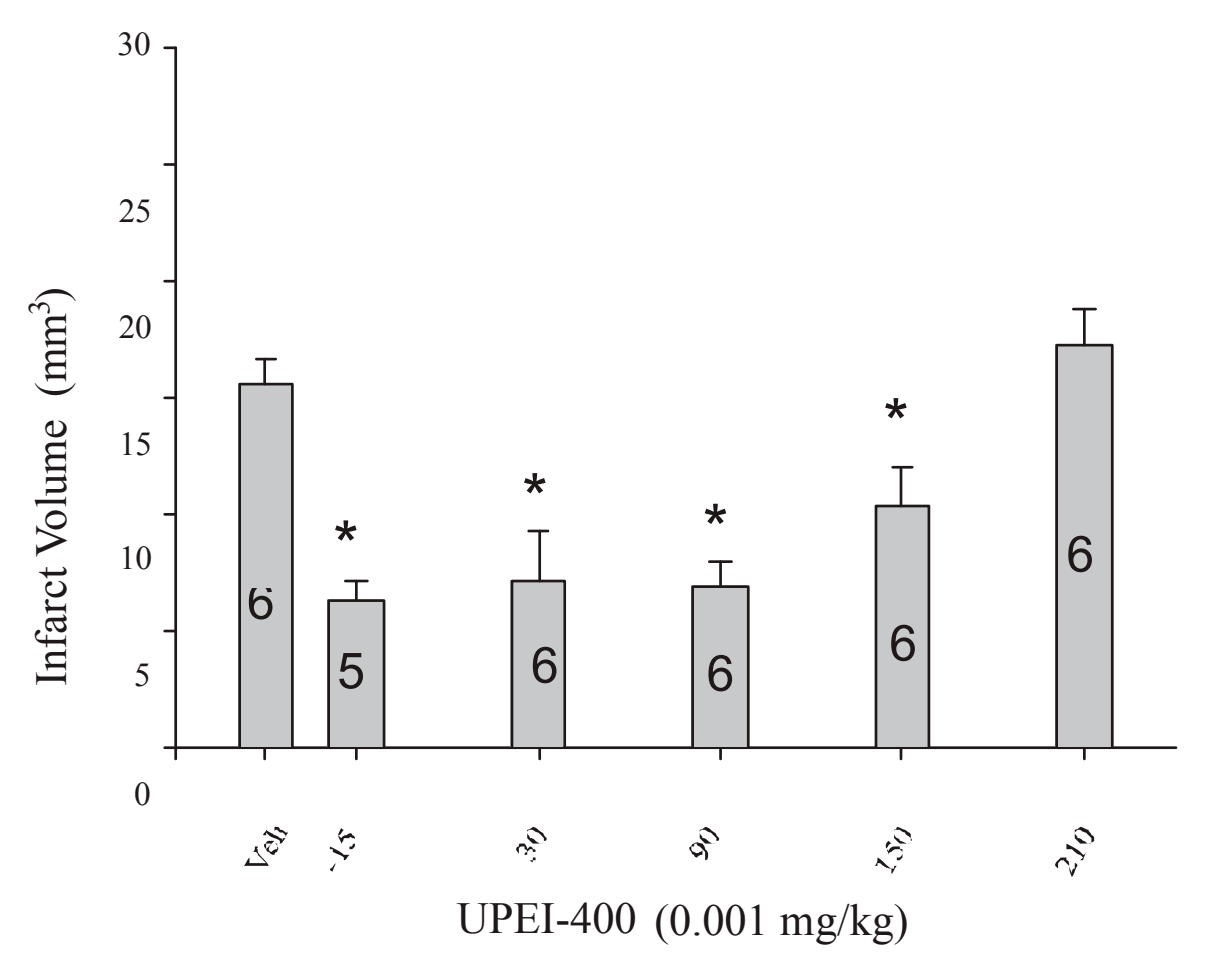

Fig 1. Dose-dependent neuroprotection observed with scopoletin.

B

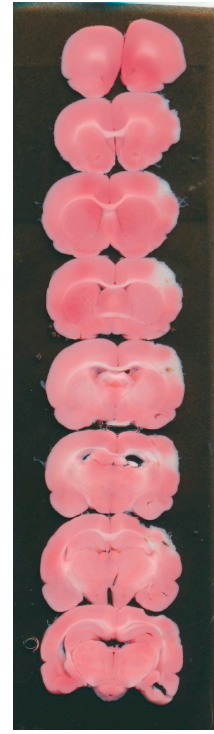


(A) Representative photomicrographs of TTC-stained, $1 \mathrm{~mm}$ thick coronal slices illustrating the extent of the infarct within the prefrontal cortex following pretreatment (30 minutes prior to MCAO; i.v.) with either vehicle (30 \% DMSO) or scopoletin $(1.0 \mathrm{mg} / \mathrm{kg})$ following ischemiareperfusion (tMCAO). (B) Bar graph summarizing the dose-response relationship between increasing doses of scopoletin and infarct volume $\left(\mathrm{mm}^{3}\right)$ calculated from TTC-stained, $1 \mathrm{~mm}$ thick coronal sections following tMCAO. Each bar represents the mean \pm S.E.M. $(n=6$ or 8/group) and * indicates significance $(p \leq 0.05)$ from the vehicle-treated control group.

The effect of pre-administration of UPEI-400 on ischemia-reperfusion (tMCAO)

Pre-administration of UPEI-400 resulted in a dose-dependent neuroprotection with 0.001 , 0.01 and $0.1 \mathrm{mg} / \mathrm{kg}$ resulting in a significant decrease in infarct volume compared to the administration of vehicle $\left(\mathrm{p} \leq 0.05 ;\right.$ Fig. $2 \mathrm{~A}_{1}$ and $\left.2 \mathrm{~B}_{1}\right)$. 
$\mathrm{A}_{1}$

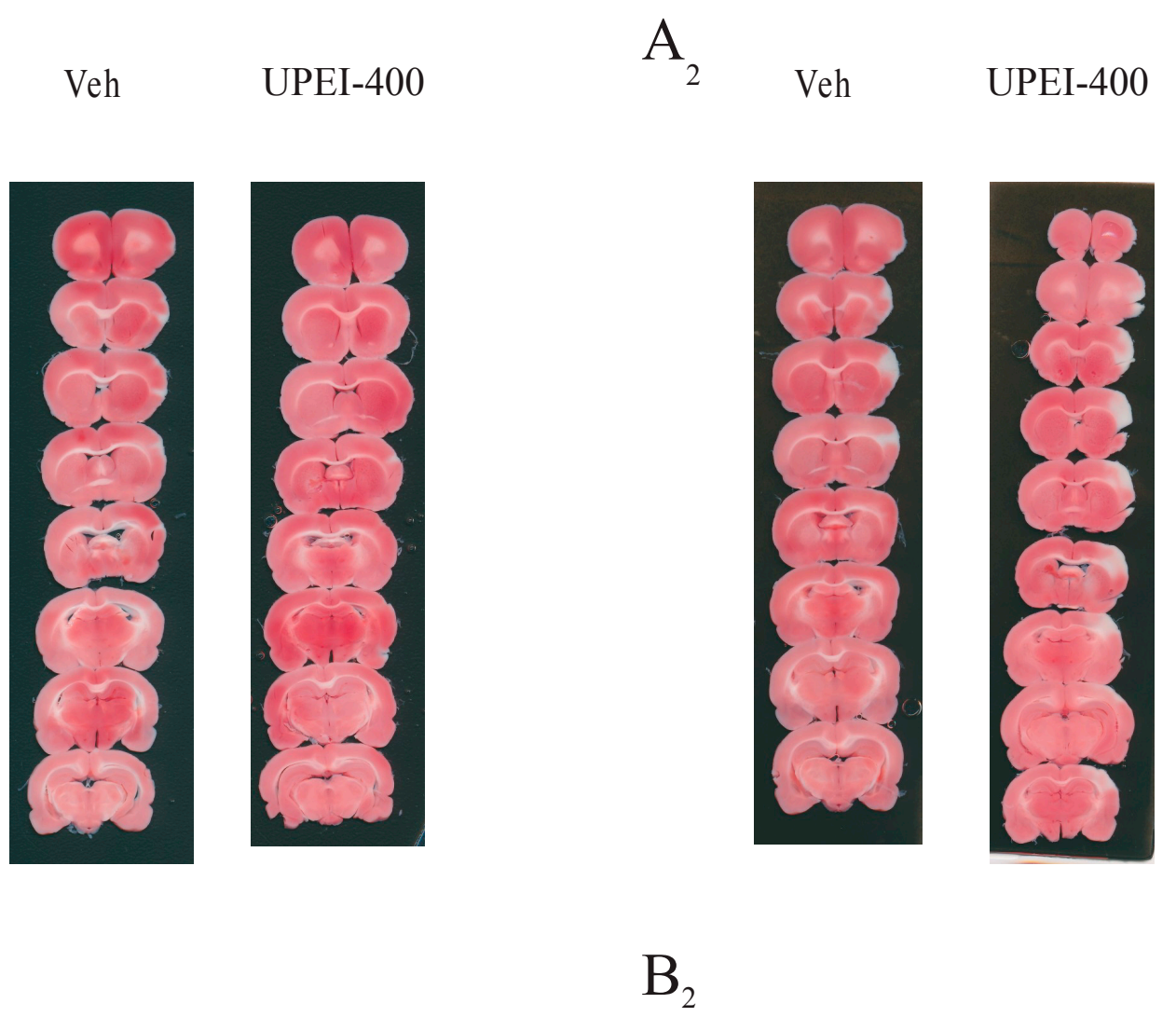

B

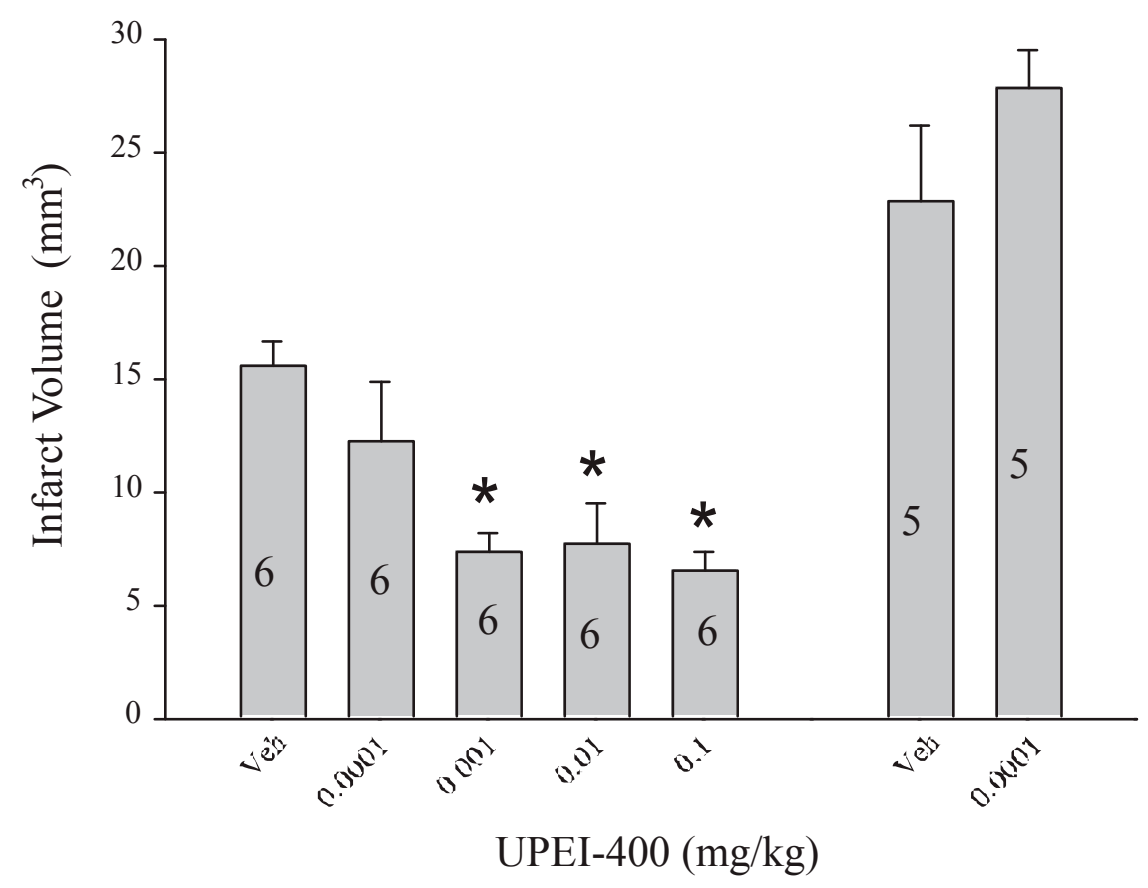

Fig 2. Dose-dependent neuroprotection observed with UPEI-400 following tMCAO, but not PMCAO. 
(A) Representative photomicrographs of TTC-stained, $1 \mathrm{~mm}$ thick coronal slices illustrating the extent of the infarct within the prefrontal cortex following pretreatment (30 minutes prior to MCAO; i.v.) with either vehicle (0.05\% DMSO) or UPEI-400 $(0.001 \mathrm{mg} / \mathrm{kg})$ following ischemia-reperfusion (tMCAO) or permanent ischemia (pMCAO). (B) Bar graph summarizing the dose-response relationship between increasing doses of UPEI-400 and infarct volume $\left(\mathrm{mm}^{3}\right)$ calculated from TTC-stained, $1 \mathrm{~mm}$ thick coronal sections following tMCAO. Also, a bar graph illustrating the effect of UPEI-400 $(0.001 \mathrm{mg} / \mathrm{kg} ; \mathrm{n}=5)$ injected 30 minutes prior to 6 hours of permanent middle cerebral artery occlusion (pMCAO) on infarct volume $\left(\mathrm{mm}^{3}\right)$ calculated from TTC-stained, $1 \mathrm{~mm}$ thick coronal sections. (vehicle $=0.05 \% \mathrm{DMSO} ; \mathrm{n}=5$ ). Each bar represents the mean \pm S.E.M. $(n=6 /$ group $)$ and * indicates significance $(p \leq 0.05)$ from the vehicle-treated control group.

The effect of pre-administration of UPEI-400 on permanent ischemia (pMCAO)

To examine the effect of UPEI-400 on ischemia-induced cell death only, administration of the optimal dose of UPEI-400 identified in the dose-response curve in Figure 2A2 $(0.001$ $\mathrm{mg} / \mathrm{kg}$ ) or vehicle were made 30 minutes prior to 6 hours of pMCAO (sutures left in place for 6 hours). UPEI-400 pre-administration did not produce significant neuroprotection when infarct volume was measured 6 hours following the start of $\mathrm{pMCAO}\left(\mathrm{p} \geq 0.05 ;\right.$ Fig. $\left.2 \mathrm{~B}_{2}\right)$.

\section{The effect of UPEI-400 administration on infarct volume when administered either during} ischemia or after the start of reperfusion

Administration of UPEI-400 $(0.001 \mathrm{mg} / \mathrm{kg}) 15$ minutes into the $30 \mathrm{~min}$ period of MCAO (15 minutes prior to the start of reperfusion; $-15 \mathrm{~min}$ on schematic) produced significant neuroprotection compared to vehicle when infarct volume was measured following 5.5 hours of reperfusion ( $\mathrm{p} \leq 0.05$; Fig. $3 \mathrm{~A})$. 
A
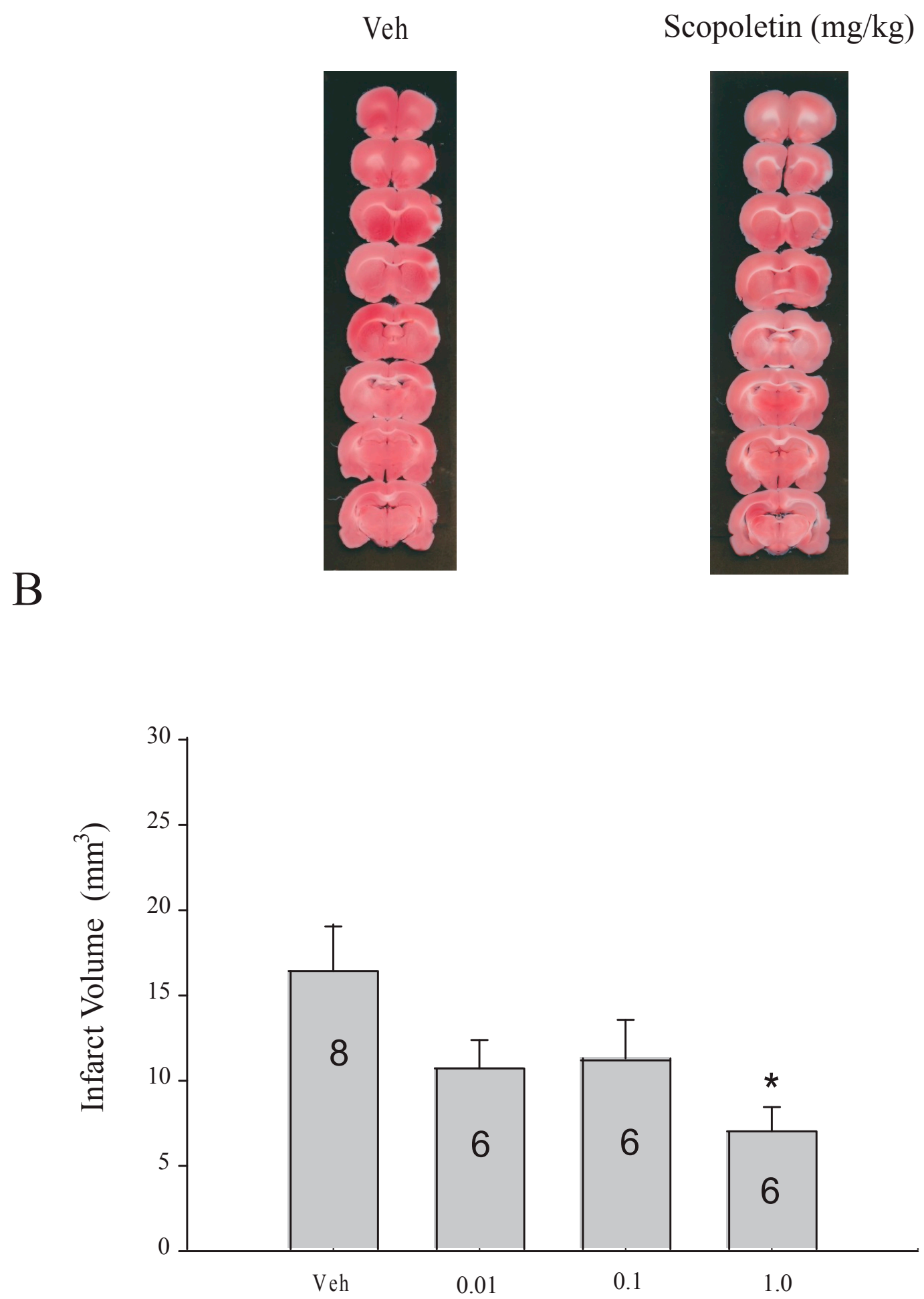

Scopoletin (mg/kg) 


\section{Fig 3. Time-course of UPEI-400 induced neuroprotection.}

(A) Representative photomicrographs of TTC-stained, $1 \mathrm{~mm}$ thick coronal slices illustrating the extent of the infarct within the prefrontal cortex following treatment with either vehicle $(0.05 \%$ DMSO) or UPEI-400 (0.001 mg/kg) 15 minutes prior to the beginning of reperfusion (-15), immediately prior to suture removal ( 0 ), or $30,90,150$, or 210 minutes ( $\mathrm{n}=5$ or $6 /$ group). (B) Effect of UPEI-400 $(0.001 \mathrm{mg} / \mathrm{kg})$ on infarct volume $\left(\mathrm{mm}^{3}\right)$ following injection 15 minutes prior to the beginning of reperfusion (-15), immediately prior to suture removal $(0)$, or $30,90,150$, or 210 minutes ( $\mathrm{n}=5$ or 6 /group) following the start of reperfusion. Each bar represents the mean \pm S.E.M. and * indicates significance $(\mathrm{p} \leq 0.05)$ from the vehicle control group $(0.05 \% \mathrm{DMSO}$; $\mathrm{n}=5$ ) injected 30 minutes prior to tMCAO.

We determined the effect of UPEI-400 on reperfusion injury only by measuring the infarct volume when the drug was only administered at 30, 90, 150 or 210 minutes after the start of reperfusion. Administration of UPEI-400 at all time points up to 150 minutes following the start of reperfusion resulted in significantly smaller infarct volumes compared to vehicle administration ( $\mathrm{p} \leq 0.05$ at each time point; Fig. 3B).

\section{The effect of UPEI-400 administration on blood pressure and heart rate}

The following experiment was designed to determine the hemodynamic effect of administration of UPEI-400 $(0.001 \mathrm{mg} / \mathrm{kg})$ on mean arterial blood pressure and heart rate for a period of 2 hours following administration. Baseline mean arterial pressure and mean heart rate prior to drug administration was $110 \pm 9 \mathrm{~mm} / \mathrm{Hg}$ and $385 \pm 16 \mathrm{bpm}$ for the vehicle $(0.05 \%$ DMSO) treated group, and, $112 \pm 5 \mathrm{~mm} / \mathrm{Hg}$ and $390 \pm 15 \mathrm{bpm}$ for the UPEI-400 $(0.001 \mathrm{mg} / \mathrm{kg})$ 
treated group. Administration of either UPEI-400 or vehicle did not significantly alter either arterial blood pressure or heart rate $(\mathrm{p} \geq 0.05)$ at any time point compared to respective preadministration (baseline) values. Further, drug administration values for blood pressure and heart rate were not significantly different from those measured in vehicle-treated animals at any time point ( $\mathrm{p} \geq 0.05$ at each time point).

\section{DISCUSSION}

In this study, we determined that UPEI-400, a chemical combination of two naturally occurring compounds, LA, and scopoletin, produced dose-dependent neuroprotection against neuronal cell death as observed in a previously validated, novel model of ischemia-reperfusion (I/R) [33]. The results demonstrated that UPEI-400 produced neuroprotection following 5.5 hours of reperfusion in a model of focal ischemia, which is restricted to the prefrontal cerebral cortex. Further, the dose of UPEI-400 required to produce significant neuroprotection $(0.001$ $\mathrm{mg} / \mathrm{kg}$ ) was 1000 fold less compared to the dose required for scopoletin alone $(1.0 \mathrm{mg} / \mathrm{kg})$, and 5000 fold less compared to the optimal neuroprotective dose of LA alone, observed previously in our lab [19]. Also, the optimal dose of UPEI-400 produced significant neuroprotection when administered 15 minutes prior to the start of reperfusion, and when administered 30, 90, and 150 minutes following the onset of reperfusion. Clinically, the paradigm of administering UPEI-400 during, and/or following the occlusion was to mimic the clinical situation in which therapy would be administered at the time a patient presents to a hospital following the onset of a stroke, or following administration of thrombolytic therapy (to prevent reperfusion injury). Interestingly, UPEI-400 did not produce neuroprotection when administered prior to a 6-hour permanent occlusive stroke (no reperfusion; pMCAO). This suggests that UPEI-400 may act to 
attenuate the oxidative stress observed following transient occlusion (I/R), whereas UPEI-400 is ineffective against the necrotic cell death primarily observed in permanent ischemia. These results also suggest that UPEI-400 provides neuroprotection against reperfusion injury alone, likely due to the scavenging of free radicals, by the attenuation of ROS production, or the inhibition of upregulated inflammation pathways.

Many studies have demonstrated anti-inflammatory and antioxidant properties of scopoletin, but to the best of our knowledge, the neuroprotective capacity of scopoletin has not been tested in an in vivo stroke model. The utility of drug therapy on reactive oxygen species and inflammation-induced growth of ischemic injury using models of reperfusion injury have been established by many labs $[2,3,34]$. In vitro studies have demonstrated that scopoletin suppressed the production of pro-inflammatory cytokines in RAW 246.7 and HMC-1 cell lines [35,36]. Scopoletin has been shown to inhibit the enzymatic activity of 5-lipoxygenase and acetylcholinesterase [6,37] and selectively inhibit MAO-b and increase dopamine levels in rodent brain tissue in vitro [38]. Scopoletin has also been shown to have antioxidant properties when tested in various antioxidant assays in vitro $[39,40]$ and is protective against glutamateinduced neurotoxicity as well as anti-cholinesterase activity in an HT22 cell line [41]. To date, most of the investigations on the therapeutic efficacy of scopoletin in vivo have focused on systemic disorders. In vivo studies have demonstrated that scopoletin is able to ameliorate the symptoms of various inflammatory, rheumatoid and digestive disorders [42,43].

Other coumarin derivatives have been shown to have antioxidant properties, which translated to neuroprotection. The coumarin derivative esculetin has been shown to reduce oxidative stress in several in vitro models $[44,45]$ and demonstrate anti-inflammatory properties in vivo [46,47]. Esculetin has been shown to be neuroprotective in a mouse model of $\mathrm{I} / \mathrm{R}$ 
following transient occlusion of the middle cerebral artery [48]. These authors also demonstrated that esculetin administration decreased cleaved caspase-3 levels, upregulated levels of Bcl-2 and downregulated levels of Bax. In addition, delayed administration of esculetin up to 4 hours following the start of reperfusion resulted in a decreased infarct size after 24 hours of reperfusion. The coumarin derivative, osthole, displayed dose-dependent neuroprotection when cultured cortical neurons were exposed to 4 hours of oxygen-glucose deprivation followed by 24 hours of reperfusion [49]. These authors demonstrated that osthole prolonged the activation of extracellular signal-regulated kinase $1 / 2($ ERK1/2) and prevented to activation of c-Jun Nterminal kinase (JNK), two members of the family of mitogen-activated protein kinases (MAPK). Osthole has also been tested an in vivo rodent stroke model. Osthole administration at the beginning of reperfusion reduced infarct volume following stroke induced by 2 hours of monofilament suture placement at the base of the MCA [7]. Osthole has also been reported to be protective against myocardial, renal and intestinal ischemia/reperfusion [50,51,52].

In the present study, UPEI-400 was not able to protect against neuronal death measured following permanent MCAO. Permanent ischemia is characterized by glutamate-induced neuronal toxicity ultimately leading to necrosis [2]. Generation of oxidative radicals and the upregulation of inflammatory pathways do not appear to play an important role in ischemic damage and subsequent cell death in an environment where there is a lack of blood flow. Therefore, anti-oxidant and anti-inflammatory agents such as scopoletin or lipoic acid, would not be effective in combating the permanent ischemia and necrotic cell death as demonstrated in the pMCAO model used in the current study.

Our results indicate that the chemical combination of scopoletin and lipoic acid produced a 1000-fold increase in I/R-induced neuroprotection compared to scopoletin alone. Similar 
results have also been presented from our laboratory. For example, using the same stroke model, the synthetic combination of lipoic acid with resveratrol (UPEI-200; [12]) produced a 200 fold increase in neuroprotection compared to resveratrol alone, a 100 fold increase in neuroprotection was found when lipoic acid was combined with apocynin (UPEI-100; [13]), and a 5 fold increase in neuroprotection was found when lipoic acid was combined with edaravone (UPEI-300; [11]). Other laboratories have also demonstrated the benefits of using chemical combinations of LA with other compounds to provide neuroprotective effects greater than either of the two compounds administered on their own. Covalent linkage of LA with ibuprofen has been demonstrated to be neuroprotective in rodent models of Alzheimer's disease where administration of the co-drug decreased the oxidative damage due to the infusion of $A \beta(1-40)$ [10]. Also, a co-drug produced by chemically linking LA with L-dopa or dopamine decreased neuronal oxidative damage associated with the administration of L-dopa or dopamine on their own [53].

Many authors have described the multiplicity of mechanisms involved in ischemia- and reperfusion-induced neuronal damage following an occlusive stroke and the difficulty in providing treatment in a clinical setting [2,54]. Therefore, drugs targeting multiple pathways might overcome this dilemma. The synthesis and development of co-drugs using biologically relevant molecules provide the ability to simultaneously target multiple pathways involved in the pathogenesis of neurological diseases, specifically, pathways involved in the initiation of oxidative stress and inflammation following reperfusion. The results presented above support the idea that the combination of antioxidant and/or anti-inflammatory compounds at subthreshold doses can produce equal or enhanced neuroprotection. The advantage of using lower doses to achieve comparable or improved therapeutic effects is to minimize side effects on other 
physiological systems as is seen in many current therapies. Our findings support the advantage of combination therapy in stroke treatment both prophylactically and even during ischemiareperfusion. UPEI-400 is a potential therapeutic candidate to protect against the negative outcomes associated with reperfusion injury.

\section{ACKNOWLEDGEMENTS}

The authors wish to thank the Atlantic Canada Opportunity Agency's Atlantic Innovation fund (file \# 199294) awarded to TMS for funding this project.

Author Contributions: TMS, BJC and MCS conceived and designed the experiments; BC and MCS performed the experiments; TMS and BJC analyzed the data; DR contributed the UPEI400 material; TMS wrote the paper.

Conflicts of Interest: The authors declare no conflict of interest.

\section{REFERENCES}

1. Mozaffarian D, Benjamin EJ, Go AS, Arnett DK, Blaha MJ, Cushman M, et al. American Heart Association Statistics Committee and Stroke Statistics Subcommittee. Circulation. 2016; 133: 447-54.

2. Ginsberg MD. Neuroprotection for ischemic stroke: past, present and future. Neuropharmacol. 2008; 55:363-389.

3. Margaill I, Plotkine M, Lerouet D. Antioxidant strategies in the treatment of strokes. Free Radic Biol Med. 2005; 39:429-443. 
4. Kang TH, Pae HO, Jeong SJ, Yoo JC, Choi BM, Jun CD, et al. Scopoletin: an inducible nitric oxide synthesis inhibitory active constituent from Artemisia feddei. Planta Med. $1999 ; 65: 400-403$.

5. Mogana R, Teng-Jin K, Wiart C. In Vitro antimicrobial, antioxidant activities and phytochemical analysis of Canarium patentinervium Miq. from Malaysia. Biotechnol Res Int. 2011;2011:768673. doi: 10.4061/2011/768673. Epub 2011 Jun 23.

6. Mogana R, Teng-Jin K, Wiart C, Anti-inflammatory, anticholinesterase, and antioxidant potential of scopoletin isolated from Canarium patentinervium Miq. (Burseraceae Kunth). Evid Based Complement Alternat Med. 2013;2013:734824. doi: 10.1155/2013/734824. Epub 2013 Jun 25.

7. Moa X, Yin W, Liu M, Ye M, Liu P, Liu J et al. Osthole, a natural coumarin improves neurobehavioral functions and reduces infarct volume and matrix metalloproteinase-9 activity after transient focal cerebral ischemia in rats. Brain Res. 2011; 1385:275-280.

8. Sun M, Hu J, Song X, Wu D, Kong L, Sun Y, et al. Coumarin derivatives protect against ischemic brain injury in rats. Eur J Med Chem. 2003; 67:39-53.

9. Bansal Y, Sliakari O. Multifunctional compounds: smart molecules for multifactorial diseases. Eur J Med Chem. 2014; 76:31-42.

10. Minnerup J, Schabitz WR. Multifunctional actions of approved and candidate stroke drugs. Neurotherapeutics. 2009; 6:43-52.

11. Connell BJ, Saleh MC, Kucukkaya I, Abd-El-Aziz AS, Khan BV, Saleh TM. UPEI-300, a conjugate of lipoic acid and edaravone mediates neuroprotection in ischemia/reperfusion. Neurosci Lett. 2014; 561:151-155. 
12. Saleh MC, Connell BJ, Rajagopal D, Khan BV, Abd-El-Aziz AS, Kucukkaya I, et al. Coadministration of resveratrol and lipoic acid or their synthetic combination, enhances neuroprotection in a rat model of ischemia/reperfusion. PLoS One. 2014; 9:1-9.

13. Connell BJ, Saleh MC, Khan BV, Rajagopal D, Saleh TM. UPEI-100, a conjugate of lipoic acid and apocynin mediates neuroprotection in a rat model of ischemia/reperfusion. Am J Physiol Regul Integr Comp Physiol. 2012; 302:R886-R895.

14. Connell BJ, Khan BV, Rajagopal D, Saleh TM. Novel neurovascular protection agents: effects of INV-155, INV-157, INV-159, and INV-161 versus lipoic acid and captopril in a rat stroke model. Cardiol Res Prac. 2012:319230. doi: 10.1155/2012/319230. Epub 2012 Jan 4.

15. Das N, Dhanawat M, Dash B, Nagarwal RC, Shrivastava SK. Codrug: an efficient approach for drug optimization. Eur J Pharm Sci. 2010; 41:571-588.

16. Sozio P, D'Aurizo E, Iannitelli A, Cataldi A, Zara S, Cantalamessa F, et al. Ibuprofen and lipoic acid diamides as potential codrugs with neuroprotective activity. Arch Pharm Chem Life Sci. 2010; 343:33-142.

17. Bilska A, Wlodek L. Lipoic acid - the drug of the future? Pharmacol Rep. 2005; 57:570577.

18. Biewengo GP, Haenen GRMM, Bast A. The pharmacology of the antioxidant lipoic acid. Gen Pharmacol. 1997; 29:315-331.

19. Connell BJ, Saleh M, Khan BV, Saleh TM. Lipoic acid protects against reperfusion injury in the early stages of cerebral ischemia. Brain Res. 2011; 1375:128-136. 
20. Richard MJ, Connell BJ, Khan BV, Saleh TM. Cellular mechanisms by which lipoic acid confers protection during the early stages of cerebral ischemia: a possible role for calcium. Neurosci Res. 2011; 69:299-307.

21. Clarke WM, Rinker LG, Lessov NS, Lowery SL, Cipolla MJ. Efficacy of antioxidant therapies in transient focal ischemia in mice. Stroke. 2001; 32:1000-1004.

22. Panigrahi M, Sadguna Y, Shivakumar BR, Kolluri SV, Roy S, Packer L, et al. alpha-Llipoic acid protects against reperfusion injury following cerebral ischemia in rats. Brain Res. 1996; 717:184-188.

23. Wolz P, Krieglstein J. Neuroprotective effects of alpha-lipoic acid and its enantiomers demonstrated in rodent models of focal cerebral ischemia. Neuropharmacol. 1996; $35: 369-375$.

24. Cao X, Phillis JW. The free radical scavenger, alpha-lipoic acid, protects against cerebral ischemia-reperfusion injury in gerbils. Free Redic Res. 1995; 23:365-370.

25. Mufherjee R, Banerjee S, Joshi N, Singh PK, Baxi D, Ramachandran AV. A combination of melatonin and alpha lipoic acid has greater cardioprotective effect than either of them singly against cadmium-induced oxidative damage. Cardiovasc Toxicol. $2011 ; 11: 78-88$.

26. Bano M, Bhatt DK. Ameliorative effect of a combination of vitamin E, vitamin C, alphalipoic acid and stilbene resveratrol on lindane induced toxicity in mice olfactory lobe and cerebrum. Indian J Exp Biol. 2010; 48:150-158.

27. Babu N, Kumar A, Singh RL. Chronic pretreatment with acetyl-L-carnitine and \pm DL- $\alpha$ lipoic acid protects against acute glutamate-induced neurotoxicity in rat brain by altering mitochondrial function. Neurotos Res. 2011; 19:319-329. 
28. Garcia-Estrada J, Gonzalez-Perez O, Gonzalez-Castaneda RE, Martinez-Contreras A, Luquin S, de la Mora PG, et al. An alpha-lipoic acid-vitamin E mixture reduces postembolism lipid peroxidation, cerebral infarction, and neurological deficit in rats. Neurosci Res. 2009; 47:219-224.

29. Sola S, Mir MQ, Cheema FA, Khan-Merchant N, Menon RG, Parthasarathy S, et al. Irbesartan and lipoic acid improve endothelial function and reduce markers of inflammation in the metabolic syndrome: results of the Irbesartan and Lipoic Acid in Endothelial Dysfunction (ISLAND) study. Circulation. 2005; 111:343-348.

30. Shotton HR, Broadbent S, Lincoln J. Prevention and partial reversal of diabetes-induced changes in enteric nerves of the rat ileum by combined treatment with alpha-lipoic acid and evening primrose oil. Auton Neurosci. 2004; 111:57-65.

31. Gonzalez-Perez O, Gonzalez-Castañeda RE, Huerta M, Luquin S, Gomez-Pinedo U, Sanchez-Almaraz E, Navarro-Ruiz A, et al. Beneficial effects of alpha-lipoic acid plus vitamin E on neurological deficit, reactive gliosis and neuronal remodeling in the penumbra of the ischemic rat brain. Neurosci Lett. 2002; 321:100-104.

32. Saleh TM, Connell BJ, Kucukkaya I, Abd-El-Aziz AS. Increasing the biological stability profile of a new chemical entity, UPEI-104, and potential use as a neuroprotectant against reperfusion-injury. Brain Sci. 2015; 5:130-143.

33. Connell BJ, Saleh TM. A novel rodent model of reperfusion injury following occlusion of the middle cerebral artery. J Neurosci Methods. 2010; 190:28-33.

34. Wang Q, Tang XN, Yenari MA. The inflammatory response in stroke. J Neuroimmunol. 2007; 184:53-68. 
35. Moon PD, Lee BH, Jeong HJ, An HJ, Park SJ, Kim HR, et al. Use of scopoletin to inhibit the production of inflammatory cytokines through inhibition of the IkappaB/NF-kappaB signal cascade in the human mast cell line HMC-1. Eur J Pharmacol. 2007; 555:218-225.

36. Kim HJ, Jang SI, Kim YJ, Chung HT, Yun YG, Kang TH, et al. Scopoletin suppresses pro-inflammatory cytokines and PGE2 from LPS-stimulated cell line, RAW 264.7 cells Fitoterapia. 2004; 75:261-266.

37. Khunnawutmanotham N, Chimnoi N, Saparpakorn P, Techasakul S. Synthesis and antiacetylcholinesterase activity of scopoletin derivatives. Bioorg Chem. 2016; 65:137-145.

38. Basu M, Mayana K, Xavier S, Balachandran S, Mishra N. Effect of scopoletin on monoamine oxidases and brain amines. Neurochem Int. 2016; 93:113-117.

39. Shaw CY, Chen CH, Hsu CC, Chen CC, Tsai YC. Antioxidant properties of scopoletin isolated from Sinomonium acutum. Phytother Res. 2003; 17:823-825.

40. Malik A, Kushnoor A, Saini V, Singhal S, Kumar S, Yadav YC. In vitro antioxidant properties of scopoletin. J Chem Pharm Res. 2011; 3:659-665.

41. Lee B, Weon JB, Eom MR, Jung YS, Ma CJ. Neuroprotective compounds of Tilia amurensis. Pharmacogn Mag. 2015; 11(Suppl 2):S303-S307.

42. Chang TN1, Deng JS, Chang YC, Lee CY, Jung-Chun L, Lee MM, et al. Ameliorative Effects of Scopoletin from Crossostephium chinensis against Inflammation Pain and Its Mechanisms in Mice. Evid Based Complement Alternat Med. 2012; 2012:1-10.

43. Yao X1, Ding Z, Xia Y, Wei Z, Luo Y, Feleder C, Dai Y. Inhibition of monosodium urate crystal-induced inflammation by scopoletin and underlying mechanisms. Int Immunopharmacol. 2012; 14:454-462. 
44. Lee SJ, Lee US, Kim WJ, Moon SK. Inhibitory effect of esculetin on migration, invasion and matrix metalloproteinase- 9 expression in TNF- $\alpha$-induced vascular smooth muscle cells. Mol Med Rep. 2011; 4:337-341.

45. Kim SH, Kang KA, Zhang R, Piao MJ, Ko DO, Wang ZH, et al. Protective effect of esculetin against oxidative stress-induced cell damage via scavenging reactive oxygen species. Acta Pharmacol Sin. 2008; 29:1319-1326.

46. Kwon OS, Choi JS, Islam MN, Kim YS, Kim HP. Inhibition of 5-lipoxygenase and skin inflammation by the aerial parts of Artemisia capillaris and its constituents. Arch Pharm Res. 2011; 34:1561-1569.

47. Witaicenis A, Seito LN, Di Stasi LC. Intestinal anti-inflammatory activity of esculetin and 4-methylesculetin in the trinitrobenzenesulphonic acid model of rat colitis. Chem Biol Interact. 2010; 186:211-218.

48. Wang C, Pei A, Chen J, Yu H, Sun ML, Liu CF, et al. A natural coumarin derivative esculetin offers neuroprotection on cerebral ischemia/reperfusion injury in mice. $\mathbf{J}$ Neurochem. 2012; 121:1007-1013.

49. Chen T, Liu W, Chao X, Qu Y, Zhang L, Luo P, et al. Neuroprotective effect of osthole against oxygen and glucose deprivation in rat cortical neurons: involvement of mitogenactivated protein kinase pathway. Neurosci. 2011; 183:203-211.

50. Dong W, Zhang Z, Liu Z, Liu H, Wang X, Bi S, et al. Protective effects of osthole, a natural derivative of coumarin, against intestinal ischemia-reperfusion injury in mice. Int J Mol Med. 2013; 31:1367-1374. 
51. Wang XY1, Dong WP, Bi SH, Pan ZG, Yu H, Wang XW, et al. Protective effects of osthole against myocardial ischemia/reperfusion injury in rats. Int J Mol Med. 2013; $32: 365-372$.

52. Zheng Y, Lu M, Ma L, Zhang S, Qiu M, Ma X. Osthole ameliorates renal ischemiareperfusion injury by inhibiting inflammatory response. Urol Int. 2013; 91:350-356.

53. Di Stefano A, Sozio P, Cocco A, Iannitelli A, Santucci E, Costa M, et al. L-dopa- and dopamine-(R)-alpha-lipoic acid conjugates as multifunctional codrugs with antioxidant properties. J Med Chem. 2006; 49:1486-1493.

54. Green AR. Pharmacological approaches to acute ischemic stroke: reperfusion certainly, neuroprotection possibly. Br J Pharmacol. 2008; 153 Suppl 1:S325-S338

(C) 2016 by the authors; licensee Preprints, Basel, Switzerland. This article is an open access article distributed under the terms and conditions of the Creative Commons by Attribution (CCBY) license (http://creativecommons.org/licenses/by/4.0/). 